KLEBERT TOSCANO DE SOUZA CINTRA

\title{
INFLUÊNCIA DO MAPEAMENTO ESTÍMULO-RESPOSTA SOBRE O EFEITO DO ESTÍMULO PRECEDENTE VISUAL
}

Dissertação apresentada ao Programa de PósGraduação em Fisiologia Humana do Instituto de Ciências Biomédicas da Universidade de São Paulo, para obtenção do Título de Mestre em Fisiologia Humana.

Área de Concentração: Fisiologia Humana

Orientador: Prof. Dr. Luiz Eduardo Ribeiro do Valle 


\section{RESUMO}

TOSCANO, K. Influência do mapeamento estímulo-resposta sobre o efeito do estímulo precedente visual. 2008. 77 f. Dissertação (mestrado em Fisiologia Humana) - Instituto de Ciências Biomédicas, Universidade de São Paulo, São Paulo, 2008.

É conhecida a capacidade do sistema nervoso de associar arbitrariamente estímulos e respostas sob demandas específicas da tarefa, no que é chamado de mapeamento estímuloresposta. Existem evidências de que este mapeamento é responsável pelo efeito inibidor encontrado em um estudo prévio sobre o tempo de reação (TR) simples, uma vez que os mesmos estímulos e respostas foram aplicados na tarefa de tempo de reação vai/não-vai, encontrando efeito facilitador. O objetivo do presente trabalho foi determinar o papel desta integração sensório-motora no efeito do estímulo precedente visual. A investigação também objetivou delimitar a estratégia relacionada com esta modulação do efeito da estimulação precedente. Para isto, foram utilizadas variações de tarefas de TR de escolha, e foram analisados os TRs e a acurácia. Encontramos uma tendência de antecipar respostas na tarefa de tempo de reação de escolha de local, devido ao aparecimento do estímulo precedente. A análise revelou uma troca de velocidade por acurácia, indicativa de alterações de critério. Uma adaptação da Teoria de Detecção de Sinais mostrou que o critério de decisão foi aumentado, evitando erros de antecipação e alarmes falsos. A tarefa de escolha de forma apresentou tempos de reação reduzidos no local previamente estimulado, sem trocas de velocidade por acurácia. Nossos resultados principais indicam que a integração entre estímulo e resposta pode interferir no efeito do estímulo precedente por meio de modulações de estratégia dependentes das características dos estímulos relevantes para a tarefa em questão.

Palavras-chave: Visão. Tempo de reação. Acurácia. Decisão. Integração sensório-motora. Critério. 


\begin{abstract}
TOSCANO, K. Influence of stimulus-response mapping on the effect of the visual prime stimulus. 2008. 77 p. Master thesis (Human Physiology) - Instituto de Ciências Biomédicas, Universidade de São Paulo, São Paulo, 2008.

It is known that the nervous system is capable of arbitrarily associate stimuli and responses under specific task demands in what is called stimulus-response mapping. There is evidence that this mapping is responsible for the inhibitory effect found in a previous study on a simple reaction time (RT) task, once the same stimuli and responses were used in go/no-go RT task and facilitatory effect was found. The aim of the present work was to determine the role of this sensory-motor integration on the effect of the visual prime stimulus. The investigation intended also to delimit the strategy related to this prime stimulus effect modulation. In order to do that, variations of choice RT task were used, and RT and accuracy data were analyzed. We found a propensity to anticipate responses in the location-based choice RT task, due to the onset of the prime stimulus. The analysis revealed a speed-accuracy trade-off, indicative of criterion shift. An adaptation of the Signal Detection Theory showed that the decision criterion was raised, avoiding anticipatory and false alarm errors. The form-based choice RT task evidenced decrease in response time in the prime stimulus location, with no speedaccuracy trade-offs. Our main results indicate that stimulus-response integration can interfere with the prime stimulus effect through strategic modulations dependent on stimuli features relevant to the task in hand.
\end{abstract}

Key-words: Vision. Reaction time. Accuracy. Decision. Sensori-motor integration. Criterion. 


\section{INTRODUÇÃO}

Os animais estão incluídos num contexto ambiental complexo e em constante mudança. Para que consigam se adaptar a este meio é necessário que realizem ações finamente adequadas no tempo e espaço que os circunda, e este conjunto de ações constitui o comportamento.

A escolha do comportamento adequado depende de processos de decisão realizados no sistema nervoso do indivíduo. Ocorrem constantemente e são resultado da atividade de diversas regiões do encéfalo que processam os estímulos do ambiente, a recompensa relacionada a cada ação, e a estratégia para lidar com tarefa em questão, integrando os estímulos às respostas (SAKAI e PASSINGHAM, 2003; SAKAI et al., 2006; FOLK, 1992; LUPIÀÑEZ, 2001).

As teorias de processamento de informação apresentam algumas etapas necessárias para o comportamento. Fuster (2004) descreveu estas etapas componentes do ciclo percepção-ação. Segundo ele, o ciclo percepção-ação é o fluxo circular de informação do ambiente para estruturas sensoriais, seguindo para estruturas motoras, que interferem no ambiente e modificam as novas entradas sensoriais. Este ciclo ocorre repetidas vezes durante o processamento do comportamento voluntário (Veja a figura 1).

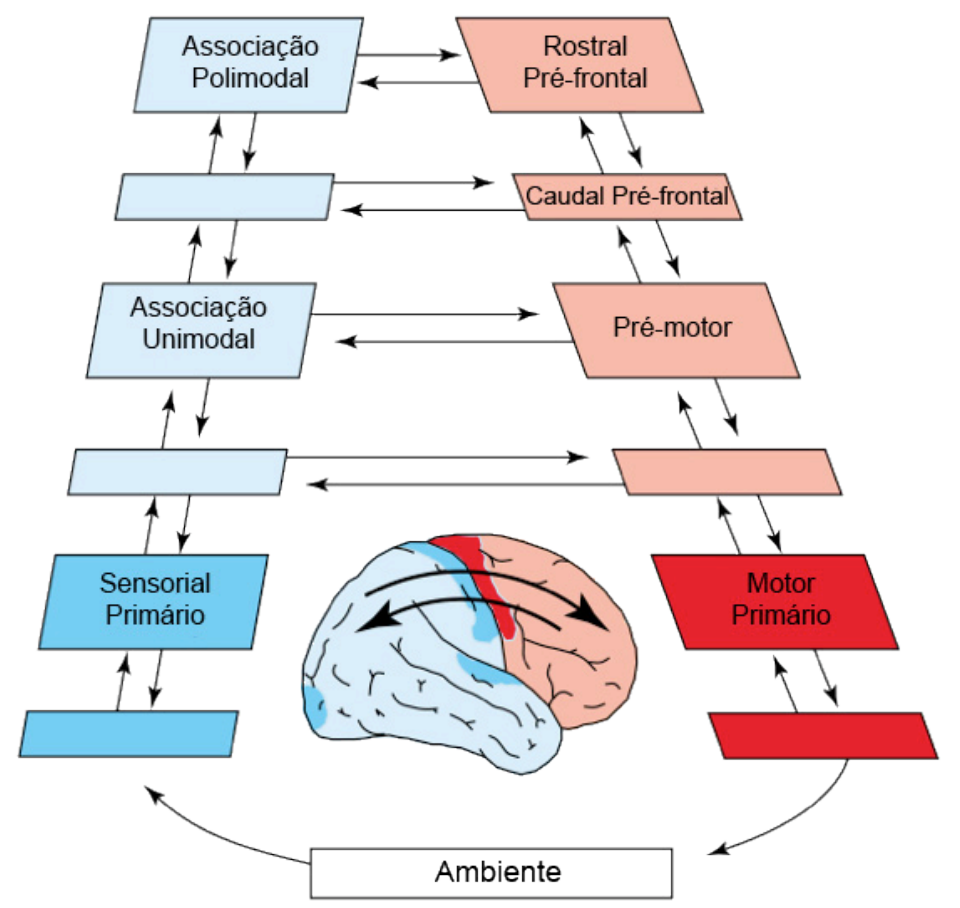

Figura 1: Substratos corticais do ciclo percepção-ação. Azul representa a parte do ciclo ligada a percepção, e o vermelho representa a parte relacionada à ação. As setas representam conexões entre as áreas corticais identificadas em macacos. (FUSTER, 2004) (modificado). 
Muitos fatores podem influenciar o processamento subjacente ao comportamento, modulando a acurácia e a latência das respostas. A psicofísica tem estudado estes fatores no intuito de compreender melhor o processamento de informações no sistema nervoso (DONDERS, 1869; STELMACH, 1982; VAN DER HEIJDEN, 1992; GESCHEIDER, 1997; HOMMEL, 2001). Um modelo experimental que foi bastante difundido pela sua simplicidade foi o desenvolvido por Posner (1980), e consiste na apresentação seqüencial de estímulos visuais numa tela de computador e na coleta das respostas dos sujeitos. São apresentados três quadrados no monitor de vídeo: um central e dois periféricos. Um dos quadrados da periferia fica com a borda mais luminosa por 100 ms, dando a sensação de piscar. Em seguida um estímulo alvo, representado por um asterisco, aparece dentro de um dos quadrados da periferia. O sujeito deve responder pressionando uma tecla ao ver o estímulo alvo.

Observa-se que os sujeitos são mais rápidos para responder ao estímulo alvo quando ele aparece na mesma posição do estímulo precedente do que na posição oposta. Posner atribuiu este efeito à mobilização da atenção pelo estímulo precedente (piscar da borda do quadrado). Supostamente o estímulo precedente facilitaria o processamento do alvo no mesmo local, e inibiria nas demais regiões do campo visual. Esta diferença nos tempos de resposta foi chamada de efeito atencional (POSNER, 1980). Como existem argumentos na literatura que atribuem este efeito a outros processos que não à atenção (PASHLER, 1999; PRINZMETAL, PARK e MCCOOL, 2005), chamaremos esta diferença de efeito positivo da estimulação precedente ${ }^{1}$.

Em estudo anterior do nosso laboratório (SQUELLA e RIBEIRO-DO-VALLE, 2003), foi comparada a influência da estimulação precedente visual em uma tarefa de tempo de reação vai/não-vai (tarefa $\mathrm{C}$ de Donders) com a influência da mesma estimulação numa tarefa de tempo de reação simples (tarefa A de Donders), seguindo um procedimento semelhante ao de Posner. Na tarefa de tempo de reação vai/não-vai (TRVNV) havia uma tela escura na qual aparecia um ponto central onde o sujeito deveria fixar o olhar. Em seguida um anel era apresentado em um dos quadrantes da tela, por $100 \mathrm{~ms}$. Este era o estímulo precedente. Em seguida, uma linha vertical no interior de um anel de mesmo tamanho que o anterior era apresentada ou no mesmo local do estímulo precedente ou no lado oposto. Este era o estímulo alvo. Quando ele aparecesse o sujeito deveria responder pressionando uma tecla com seu dedo indicador direito tão $\operatorname{logo}$ o visse. Caso fosse apresentado um estímulo

\footnotetext{
${ }^{1} \mathrm{O}$ efeito do estímulo precedente é calculado pela subtração da média das medianas dos tempos de reação na posição oposta menos a média das medianas dos tempos de reação na posição mesma. O resultado pode ser um valor positivo ou negativo, caracterizando uma facilitação ou inibição, respectivamente, do total dos processos até a resposta.
} 
alvo negativo, constituído de um anel menor dentro de outro anel, o sujeito não deveria emitir resposta.

Ao executar esta tarefa, os sujeitos foram mais rápidos para responder quando os estímulos precedente e alvo apareceram na mesma posição que quando em posições opostas, de acordo com o esperado.

A tarefa de tempo de reação simples (TRS) foi como a de TRVNV, sendo a única diferença não haver o estímulo alvo negativo. Surpreendentemente, um efeito oposto foi observado. Os sujeitos foram mais lentos para responder quando os estímulos precedente e alvo apareceram na mesma posição. Este retardo na resposta acontecia com intervalos muito curtos entre o estímulo precedente e o alvo (100 ms).

Deve-se notar que as características físicas dos estímulos visuais, como luminância, excentricidade e forma do estímulo alvo, intervalos temporais entre os estímulos e ausência de demarcações de posições relevantes na tela do monitor de vídeo não diferiram entre as duas situações, logo, os fatores relacionados aos componentes sensoriais podem ser descartados. As características relacionadas ao componente motor também não foram modificadas havendo os mesmos intervalos de tempo variáveis entre as tentativas, evitando a preparação no tempo, e somente uma resposta a ser preparada ou inibida (KLAPP, 2003).

Uma vez que os componentes sensoriais e motores separadamente não se mostraram suficientes para explicar os dados observados nas tarefas acima, decidimos investigar novamente a questão, desta vez sob o ponto de vista da integração sensório-motora.

Somos possuidores de uma grande capacidade de fazer associações arbitrárias entre estímulos e ações, o que é conhecido como mapeamento estímulo-resposta. Regiões cerebrais como a área intraparietal lateral, campo ocular frontal, e o campo ocular suplementar são apontadas como as regiões corticais responsáveis por fazer estas associações, aliados à função dos núcleos da base e áreas hipocampais (COE et al, 2002; WISE e MURRAY, 2000; ORISTAGLIO et al., 2006; GOTTLIEB, 2007).

As bases neurofisiológicas para isso consistem na integração de múltiplas variáveis comportamentais, que incluem a resposta motora específica, a recompensa relacionada ao desempenho, e a decodificação do objeto relevante dentro de regras abstratas. Foi observado que isto ocorre em diversas tarefas e associado a respostas motoras distintas (SATO et al., 2001; COE et al., 2002; SCHALL, 2003; SATO e SCHALL, 2003; ORISTAGLIO et al., 2006; GOTTLIEB, 2007). 
Para que estas informações sejam integradas, é necessário que a informação que chega às áreas em questão seja acumulada e em seguida a comparação entre as evidências para uma ou outra resposta e recompensa associada sejam comparadas. As células destas áreas associativas possuem um mecanismo de acúmulo de informação resultado da atividade de receptores de glutamato NMDA. Eles possibilitam a integração temporal de informação incidente por meio do acúmulo de cálcio intracelular, que permite às células interligadas uma situação de dupla estabilidade. (LO e WANG, 2006; WONG e WANG, 2006; SAKAI, OKAMOTO, FUKAI, 2006).

Além de mecanismos neurais para acúmulo de informação, foi proposto um mecanismo intracelular que independe de sinapses para manter o nível de disparo de um neurônio - o que pode ser interpretado como um acúmulo de informação em nível celular. Ele se baseia no pressuposto que os íons de cálcio não são permeáveis às membranas intracelulares. Desta forma, haveria diferenças de concentração do íon, que poderiam formar ondas que codificariam o resultado de integrações temporais dos últimos inputs para o modelo neuronal. A informação armazenada pela concentração de cálcio pode ser traduzida em índice de disparo, por meio de correntes catiônicas cálcio-dependentes distribuídas uniformemente ao longo do dendrito (SAKAI, OKAMOTO, FUKAI, 2006).

Com o aumento da atividade nas células que compõem as redes das áreas associativas em questão, a atividade cresce até chegar a um ponto onde excede um limiar e ocorre a ativação de áreas motoras, executando assim a resposta. Isto está de acordo com o modelo de difusão, que se baseia na premissa de que a representação dos estímulos no sistema nervoso é variável e ruidosa. Por isso deve ser acumulada informação até que uma quantidade de evidências, o critério, seja obtida (SPERLING e DOSHER, 1986; RATCLIFF e SMITH, 2004; RATCLIFF, 1987, 2002, 2006). Para mais informações, veja o anexo A.

Este modelo nos fornece informações muito importantes na busca pela explicação do comportamento e da integração estímulo-resposta, pois no estabelecimento do critério, define que resposta será executada, como resultado do acúmulo de informações sensoriais; e no tempo necessário para atingir o critério, define o tempo de reação.

Estudos na literatura (IVANOFF e KLEIN, 2004; LUPIÀÑEZ et al., 2005) compararam uma situação onde houve alta freqüência de ocorrência de um estímulo associado a uma resposta (mapeamento estímulo-resposta), com uma situação onde esta freqüência era baixa. Foi observado que na situação de mapeamento estímulo-resposta mais freqüente, a estimulação precedente promoveu aumento nos tempos de reação. Este retardo nas respostas 
ocorreu com curtos intervalos entre os estímulos precedente e alvo, e se manteve por centenas de milissegundos - o mesmo padrão encontrado nos experimentos de TRS de nosso laboratório (SQUELLA e RIBEIRO-DO-VALLE, 2003).

Caso este resultado esteja relacionado à inibição dos recursos atencionais, é esperado que o sujeito tenha um menor desempenho também na acurácia de suas respostas, pois o processamento sensorial será desprivilegiado. Entretanto, estudos semelhantes demonstraram que a acurácia dos sujeitos era maior na posição previamente estimulada do que na posição oposta (IVANOFF e KLEIN, 2001, 2003, 2006). O aumento da acurácia às custas de retardo no tempo de reação, conhecido como troca velocidade-acurácia, é característico de aumento de critério. Com um critério aumentado, mais informação é necessária para que seja alcançado o limiar de decisão, e menos erros são cometidos (SPERLING e DOSHER, 1986; RATCLIFF e SMITH, 2004; RATCLIFF, 2006).

A teoria de detecção de sinais (TDS) é de grande valia para análise de erros em tarefas psicofísicas, pois é uma abordagem geral para medida de performance. Ela se aplica quando um sistema deve detectar um sinal em meio a ruído. Sua importância está na capacidade de investigar separadamente o componente sensorial do sistema e de decisão da resposta por meio das medidas de $d$ ' e de critério $^{2}$, provendo um meio de análise da estrutura das decisões do observador (GREEN e SWETS, 1966; SPERLING e DOSHER, 1986; MACMILLAN e CREELMAN, 2005). O anexo B oferece mais informações a respeito da teoria.

Se aplicada a TDS aos dados dos experimentos de Squella e Ribeiro-do-Valle (2003), poderiam ser apresentadas medidas quantitativas da troca velocidade-acurácia. No entanto, existem limitações metodológicas para fazê-lo. Em primeiro lugar, a TDS é voltada para análise em tarefas de acurácia, e não de tempo de reação (MACMILLAN e CREELMAN, 1986). Em segundo lugar, não é possível separar as condições de erro necessárias para a aplicação da teoria em tarefas de TRS e TRVNV, pois só existe um tipo de resposta a ser executada pelo voluntário. Neste caso, não há como separar respostas

\footnotetext{
${ }^{2} \mathrm{O}$ critério existe em qualquer situação onde se busca separar o sinal do ruído por meio de decisão estatística. No caso de resposta a estímulo visual, vai desde a retina, até o córtex visual primário, áreas integrativas de onde segue para os córtices motores, cada um com sua relação entre sinal e ruído do sistema. Nosso enfoque de critério está na decisão da resposta a ser executada, e não à parte de construção interna da representação dos estímulos externos. As áreas onde esta decisão ocorre são o córtex intraparietal lateral, campo ocular frontal, e o campo ocular suplementar, recebendo modulações de áreas relacionadas a recompensa: o córtex cingulado anterior e o córtex orbitofrontal (COE et al, 2002; WISE \& MURRAY, 2000; ORISTAGLIO et al., 2006; RUDEBECK et al., 2006; GOTTLIEB, 2007; RUSHWORTH et al., 2007).
} 
decorrentes do aparecimento do alvo de respostas decorrentes do aparecimento do estímulo precedente. (MACMILLAN e CREELMAN, 1986; IVANOFF e KLEIN, 2006). Por esta razão utilizamos tarefas de tempo de reação de escolha. Este tipo de tarefa permite analisar os dados de acurácia, discriminando respostas legítimas ao alvo de alarmes falsos. Se comprovarmos que o estímulo precedente pode desencadear respostas que devem ocorrer apenas para o alvo, podemos supor que o aumento dos tempos de reação na tarefa de TRS seja decorrente de alterações de estratégia dos voluntários (aumento de critério) para melhorar o desempenho na acurácia. 


\section{CONCLUSÃO}

Os resultados de tempo de reação, associados aos de acurácia, indicam que o mapeamento estímulo-resposta pode interferir nos efeitos do estímulo precedente visual. Esta interferência é resultado de alterações da estratégia de decisão, por modificação do critério de resposta. 


\section{REFERÊNCIAS BIBLIOGRÁFICAS ${ }^{6}$}

ADOLPHS, R; Emotional vision. Nature Neuroscience, v. 7, n. 11, 2004.

BAR, M. Visual objects in context. Nature Reviews - Neuroscience, v. 5, p. 617-629, 2004.

BADLER, J. B.; HEINEN, S. Anticipatory movement timing using prediction and external cues. Journal of Neuroscience, v. 26, n. 17, p. 4519-4525, 2006.

BARCELÓ, F.; SUWAZONO, S.; KNIGHT, R. T. Prefrontal modulation of visual processing in humans. Nature Neuroscience, v. 3, n. 4, p. 399-403, 2000.

CASTEL, A. D.; PRATT, J.; CHASTEEN, A. L.; SCIALFA, C. T. Examining task difficulty and the time course of inhibition of return: detecting perceptually degraded targets. Canadian Journal of Experimental Psychology, v. 59, n. 2, p. 90-98, 2005.

CAVANAUGH, J.; WURTZ, R. Subcortical modulation of attention counters change blindness. Journal of Neuroscience, v. 24, n. 50, p. 11236-11243, 2004.

CHEN, Z.; CAVE, K. R. When does visual attention select all features of a distractor? Journal of Experimental Psychology: Human Perception and Performance, v. 32, n. 6, p. 1452-1464, 2006.

CHUN, R.; MAROIS, R. The dark side of visual attention. Current Opinion in Neurobiology, v. 12, p. 184-189, 2002.

COE, B.; TOMIHARA, K.; MATSUZAWA, M.; HIKOSAKA, O. Visual and anticipatory bias in three cortical eye fields of the monkey during an adaptive decision-making task. Journal of Neuroscience, v. 22, n. 12, p. 5081-5090, 2002.

CONNOR, C. E.; PREDDIE, D. C.; GALLANT, J. L.; ESSEN, D. C. V. Spatial attention effects in macaque area V4. Journal of Neuroscience, v. 17, n. 9, p. 3201-3214, 1997.

COULL, J. T. fMRI studies of temporal attention: allocating attention within, or towards, time. Cognitive Brain Research, v. 21, p. 216-226, 2004.

CORBETTA, M.; SCHULMAN, G. L. Control of goal-directed and stimulus-driven attention in the brain. Nature Reviews - Neuroscience, v. 3, p. 201-215, 2002.

EIMER, M. A ERP study of sustained spatial attention to stimulus eccentricity. Biological Psychology, v. 52, p. 205-220, 2000.

\footnotetext{
6 ASSOCIAÇÃO BRASILEIRA DE NORMAS TÉCNICAS. NBR 6023: Informação e documentação: referências: elaboração. Rio de Janeiro, 2002.

NATIONAL LIBRARY OF MEDICINE. List of journals in Index Medicus. 2006. Available from: http://www.nbci.nlm.nih.gov/entrez/; http://www.nlm.nih.gov/tsd/serials/lji.html.
} 
EYSENK, M. W.; KEANE, M. T.; Limitações da atenção e do desempenho. In: Psicologia cognitiva: um manual introdutório. Porto Alegre: Artes Médicas, 1994.

FOLK. C. L. REMINGTON, R.W. JOHNSTON, J. C. Involuntary covert orienting is contingent on attentional control setting. Journal of Experimental Psychology: human perception and performance, v. 18, p. 1030-1044, 1992.

FU, S.; GREENWOOD, P. M.; PARASURAMAN, R. Brain mechanisms of involuntary visuospatial attention: an event-related potential study. Human Brain Mapping, v. 25, p. 378$390,2005$.

FUSTER, J. M. upper processing stages of the perception-action cycle. TRENDS in Cognitive Sciences, v. 8, n. 4, 2004.

HANSEN, S.; ELLIOTT, D. Nomadic inhibition of attention and motor responses. Human Movement Science, v. 24, p. 744-759, 2005.

HICKEY, C.; McDONALD, J.; THEEUWES, J. Electrophysiological Evidence of the Capture of Visual Attention. Journal of Cognitive Neuroscience, v. 18, n. 4, p. 604-613, 2006.

HOMMEL, B. Feature integration across perception and action: event files affect response choice. Psychological Research, 2005. DOI 10.1007/s00426-005-0035-1.

HOMMEL, B. How much attention does an event file need? Journal of Experimental Psychology: Human Perception and Performance, v. 31, n. 5, p. 1067-1082, 2005.

HOMMEL, B.; SCHNEIDER, W. X. Visual attention and manual response selection: Distinct mechanisms operating on the same codes. Visual Cognition, v. 9, p. 392-420, 2002.

GAZZANIGA, M. S.; IVRY, R. B.; MANGUN, G. R. Attention and selective perception. In: 1988 .

Cognitive Neuroscience: the biology of the mind. New York - London: W. W. Norton,

GOTTLIEB, J. From thought to action: the parietal cortex as a bridge between perception, action and cognition. Neuron Review, n. 53, p. 9-16, 2007.

IPATA, A. E.; GEE, A. L.; GOTTLIEB, J.; BISLEY, J. W.; GOLDBERG, M. E. LIP Responses to a popout stimulus are reduced if it is overtly ignored. Nature Neuroscience, v. 9, n. 8, 2006.

IVANOFF, J.; KLEIN, R. M. The presence of a nonresponding effector increases inhibition of return. Psychonomic Bulletin \& Review, v. 8, n. 2, p. 307-314, 2001.

IVANOFF, J.; KLEIN, R. M. Orienting of attention without awareness is affected by measurements-induced attentional control settings. Journal of Vision, v. 3, p. 32-40, 2003.

IVANOFF, J.; KLEIN, R. M. Stimulus-response probability and inhibition of return. Psychonomic Bulletin \& Review, v. 11, n. 3, p. 542-550, 2004. 
IVANOFF, J.; KLEIN, R. M.; LUPIÁÑEZ, J. Inhibition of return interacts with the Simon effect: an omnibus analysis and its implications. Perception \& Psychophysics, v. 64, n. 2, p. 318-327, 2002.

KANWISHER, N.; WOJCIULIK, E. Visual attention: insights from brain imaging. Nature Reviews - Neuroscience, v.1, p. 91-100, 2000.

KLAPP, S. T. Reaction time analysis of two types of motor preparation for speech articulation: action as a sequence of chuncks. Journal of Motor Behavior, v. 35, n. 2, p. 135$150,2003$.

KLEIN, R. M. Inhibition of return. Trends in Cognitive Sciences, vol. 4, n. 4, 2000.

KOK, A.; RIDDERINKHOF, R.; ULLSPERGER, M. The control of attention and action: current research and future developments. Brain Research, v. 1105, p. 1-6, 2006. DOI: 10.1016/j.brainres.2006.03.027.

LO, C.; WANG, X. Cortico-basal ganglia circuit mechanism for a decision threshold in reaction time tasks. Nature Neuroscience, v. 9, n. 7, 2006.

LU, L.; LESMES, A. L.; DOSHER, A. B. Spatial attention excludes external noise at the target location. Journal of Vision, v. 22, p. 312-323, 2002.

LUCK, S. J.; THOMAS, S. J. What variety of attention is automatically captured by peripheral cues? Perception \& Psychophysics, v. 61, n. 7, p. 1424-1435, 1999.

LUCK, S. J.; WOODMAN, G. F.; VOGEL, E. K. Event-related potential studies of attention. Trends in Cognitive Sciences, v. 4, n. 11, 2000.

LUPIÁÑEZ, J.; MILAN, E. G.; TORNAY, F. J.; MADRID, E.; TUDELA, P. Does IOR occur in discrimination tasks? yes, it does, but later. Perception \& Psychophysics, v. 59, n. 8, p. 1241-1254, 1997.

LUPIÁÑEZ, J.; MILLIKEN, B.; SOLANO, C.; WEAVER, B.; TIPPER, S. P. On the strategic modulation of the time course of facilitation and inhibition of return. The Quarterly Journal of Experimental Psychology, v. 54A, n. 3, p. 753-773, 2001.

LUPIÁÑEZ, J.; RUZ, M.; FUNES, M. J.; MILLIKEN, B. The manifestation of attentional capture: facilitation of IOR depending on task demands. Psychological Research, 2005. DOI 10.1007/s--426-005-0037-z.

MACALUSO, E.; DRIVER, J. Multisensory spatial interactions: a window onto functional integrations in the human brain. TRENDS in Neurosciences, v. 28, n. 5, 2005.

MAROIS, R.; IVANOFF, J. Capacity limits of information processing in the brain. TRENDS in Cognitive Sciences, v. 9, n. 6, p. 296-305, 2005.

MCCOY, A. N.; PLATT, M. L. Expectations and outcomes: decision-making in the primate brain. Journal of Comparative Physiology A, v. 191, p. 201-211, 2005. DOI 10.1007/s00359004-0565-9. 
MÜLLER, H. J.; FINDLAY, J. M. Sensitivity and criterion effects in the spatial cuing of visual attention. Perception \& Psychophysics, v. 42, n. 4, p. 383-399, 1987.

MURRAY, I. J.; PLAINIS, S. Contrast coding and magno/parvo segregation revealed in reaction time studies. Vision Research, v. 43, p. 2707-2719, 2003.

NEUMANN, O.; SCHARLAU, I. Visual attention and the mechanism of metacontrast. Psychological Research, v. 71, p. 626-633, 2007.

NIEUWENHUIS, S.; YEUNG, N. Neural mechanisms of attention and control: losing our inhibitions? Nature Neuroscience, v. 8, n. 12, p. 1631-1633, 2005.

ORISTAGLIO, J.; SCHNEIDER, D. M.; BALAN, P. F.; GOTTLIEB, J. Integration of visuospatial and effector information during symbolically cued limb movements in monkey lateral intraparietal area. Journal of Neuroscience, v. 26, n. 32, p. 8310-8319, 2006.

PALMER, J.; HUK, A. C.; SHADLEN, M. N. The effect of stimulus strength on the speed and accuracy of a perceptual decision. Journal of Vision, v. 5, 376-404, 2005.

PASHLER, H. E.; The Psychology of Attention, MIT Press, 1999.

PASSINGHAM, R. E.; TONI, I. Contrasting the dorsal and ventral visual systems: guidance of movement versus decision making. NeuroImage, v. 14, p. S125-S131, 2001.

PESTILLI, F.; CARRASCO, M.; Attention enhances contrast sensitivity at cued and impairs it at uncued locations. Vision Research, v. 45, p. 1867-1875, 2005.

POSNER, M. I. Orienting of attention. The 7th Sir F. C. Bartlett lecture. Quarterly Journal of Experimental Psychology, v. 32, p. 3-25, 1980.

PRAAMSTRA, P.; OOSTENVELD, R. Attention and movement-related motor cortex activation: a high-density EEG study of spatial stimulus-response compatibility. Cognitive Brain Research, v. 16, p. 309-322, 2003.

PRATT, J.; KINGSTON, A.; KHOE, W. Inhibition of return in location- and indentity-based choice decision tasks. Perception \& Psychophysics, v. 59, n. 6, p. 964-971, 1997.

PRIME, D. J.; WARD, L. M. Cortical expressions of inhibition of return. Brain Research, v. 1072, p. 161-174, 2006.

PRINZMETAL, W.; PARK, S.; MCCOOL, C. Attention: reaction time and accuracy reveal different mechanisms. Journal of Experimental Psychology: general, v. 134, n. 1, p. 73-92, 2005 .

RATCLIFF, R. A diffusion model account of response time and accuracy in a brightness discrimination task: fitting real data and failing to fit fake but plausible data. Psychonomic Bulletin \& Review, v. 9, n. 2, p. 278-291, 2002. 
SCHALL, J. D. Neural basis of deciding, choosing and acting. Nature Reviews Neuroscience, v. 2, p. 33-42, 2001.

SCHALL, J. D. Decision making: neural correlates of response time. Current Biology, v. 12, p. R800-R801, 2002.

SCHALL, J. D. Neural correlates of decision processes: neural and mental chronometry. Current Opinion in Neurobiology, v. 13, p. 182-186, 2003.

SCHALL, J. D. Decision making. Current Biology, v. 15, n. 1, 2005.

SHIPP, S. The brain circuitry of attention. TRENDS in Cognitive Sciences, v. 8, n. 5, p. 223230, 2004.

SMITH, P. L.; RATCLIFF, R. Psychology and neurobiology of simple decisions. TRENDS in Neurosciences, v. 27, n. 3, 2004.

SPERLING, G.; DOSHER, B. A. Strategy and optimization in human information processing. In: BOFF, K. R.; KAUFMAN, L.; THOMAS, J. P. Handbook of Perception and Human Performance, New York: John Wiley \& Sons, 1986. v. 1.

SQUELLA, S. A. F.; RIBEIRO-DO-VALLE, L. E. Priming effects of a peripheral visual stimulus in simple and go/no-go tasks. Brazilian Journal of Medical and Biological Research, v. 36, p. 247-261, 2003.

STELMACH, G. E. Information-processing framework for understanding human motor behavior. In: KELSO, J. A. S., Human Motor Behavior: an introduction. New York: Lawrence-Erlbaum, 1982. p. 63-92.

STERNBERG, S. The discovery of processing stages: extensions of Donders' method. Acta Psychologica 30 Attention and Performance, v. 11, p. 276-315, 1969.

STOET, G.; SNYDER, L. H.; Correlates of stimulus-response congruence in the posterior parietal cortex. Journal of Cognitive Neuroscience, v. 19, n. 2, p. 194-203, 2007.

TASSINARI, G.; AGLIOTI, S.; CHELAZZI, L.; PERU, A.; BERLUCHI, G. Do peripheral non-informative cues induce early facilitation of Target Detection? Vision Research, v. 34, n. 2, p. 174-189, 1994.

VAN DER HEIJDEN. Selective attention in vision. International Library of Psychology: Routledge, 1992.

VAN DER LUBBE, R. O.; VOGEL, R. O.; POSTMA, A. Different effects of exogenous cues in a visual detection and discrimination task: delayed attention withdrawal and/or speeded motor inhibition? Journal of Cognitive Neuroscience, v.17, n.12, p. 1829-1840, 2005.

VAN DER LUBBE, H. J.; MAARTEN M. H.; BEKKER, M. E.; POSTMA, A. Taskdependent exogenous cuing effects depend on cue modality. Psychophysiology, v.43, p.145149, 2006. 
RATCLIFF, R.; SMITH, P. L. A comparison of sequential sampling models for two-choice reaction time. Psychological Review, v. 111, n. 2, p. 333-367, 2004.

RATCLIFF, R. Modeling response signal and response time data. Cognitive Psychology, v. 53, p. 195-237, 2006.

RAZ, A.; BUHLE, J. Typologies of attentional networks. Nature Reviews - Neuroscience, v. 7, p. 367-379, 2006.

REMINGTON, R. W.; JOHNSTON, J. C.; YANTIS, S. Involuntary attentional capture by abrupt onsets. Perception \& Psychophysics, v. 51, n. 3, p. 279-290, 1992.

RESS, D.; BACKUS, B.; HEEGER, D. Activity in primary visual cortex predicts performance in a visual detection task. Nature Neuroscience, v. 3, n. 9, 2000.

ROELFSEMA, P. R. Elemental operations in vision. TRENDS in Cognitive Sciences, v. 9, n. 5, p. 226-233, 2005.

RUDEBECK, P. H.; WALTON, M. E.; SMYTH, A. N.; BANNERMAN, D. M.; RUSHWORTH, M. F. S. Separate neural pathways process different decision costs. Nature Neuroscience, v. 9, n. 9, 2006.

RUSHWORTH, M. F. S.; BEHRENS, T. E. J.; RUDEBECK, P. H.; WALTON, M. E. Contrasting roles for cingulate and orbitofrontal cortex in decision and social behaviour. TRENDS in Cognitive Sciences, v. 11, n. 4, 2007.

RUSHWORTH, M. F. S.; WALTON, M. E.; KENNERLEY, S. W.; BANNERMAN, D. M. Action sets and decision in the medial frontal cortex. TRENDS in Cognitive Sciences, v. 8, n. 9, 2004.

SAKAI, K.; PASSINGHAM, R. E. Prefrontal interactions reflect future task operations. Nature Neuroscience, v. 6, n. 1, 2003.

SAKAI, Y.; OKAMOTO, H.; FUKAI, T. Computational algorithms and neuronal network models underlying decision processes. Neural Networks, 19, p. 1091-1105, 2006.

SATO, T. R.; SCHALL, J. D. Effects of stimulus-response compatibility on neural selection in frontal eye field. Neuron, v. 38, p. 637-648, 2003.

SATO, T.; MURTHY, A.; THOMPSON, K. G.; SCHALL, J. D. Search efficiency but not response interference affects visual selection in frontal eye field. Neuron, v. 30, p. 583-591, 2001.

SCERIF, G.; WORDEN, M. S.; DAVIDSON, M.; SEIGER, L.; CASEY, B. J. Context modulates early stimulus processing when resolving stimulus-response conflict. Journal of Cognitive Neuroscience, v. 18, n. 5, p. 781-792, 2006.

SCHALL, J. D. Decision making: from sensory evidence to a motor command. Current Biology, v. 10, p. 404-406, 2000. 
VOSSEL, S.; THIEL, C. M.; FINK, G. R. Cue validity modulates the neural correlates of covert endogenous orienting of attention in parietal and frontal cortex. NeuroImage, v. 32, p. 1257-1264, 2003.

VUILLEUMIER, P. How brains beware: neural mechanisms of emotional attention. TRENDS in Cognitive Sciences, v.9, n. 12, p. 585-594, 2005.

WANG, C.; ULBERT, I.; SCHOMER, D.; MARINKOVIC, K.; HALGREN, E. Responses of human anterior cingulate cortex microdomains to error detection, conflict monitoring, stimulus-response mapping, familiarity, and orienting. Journal of Neuroscience, v. 25, n. 3, p. 604-613, 2005.

WASZAK, F.; WASCHER, E.; KELLER, P.; KOCH, I.; ASCHERSLEBEN, G.; ROSENBAUM, D. A.; PRINZ, W. Intention-based and stimulus-based mechanisms in action selection. Experimental Brain Research, v. 162, p. 346-356, 2005.

WISE, S. P.; MURRAY, E. A. Arbitrary associations between antecedents and actions. TRENDS in Neuroscience, v. 23, n. 6, 2000.

WOJCIULIK, E.; KANWISHER, N. The generality of parietal involvement in visual attention. Neuron, v. 23, p. 747-764, 1999.

WONG, K.; WANG, X. A recurrent network mechanism of time integration in perceptual decisions. Journal of Neuroscience, v. 26, n. 4, p. 1314-1328, 2006.

YANTIS, S.; SERENCES, J. Cortical mechanisms of space-based and object-based attentional control. Current Opinion in Neurobiology, v. 13, p. 187-193, 2003. 